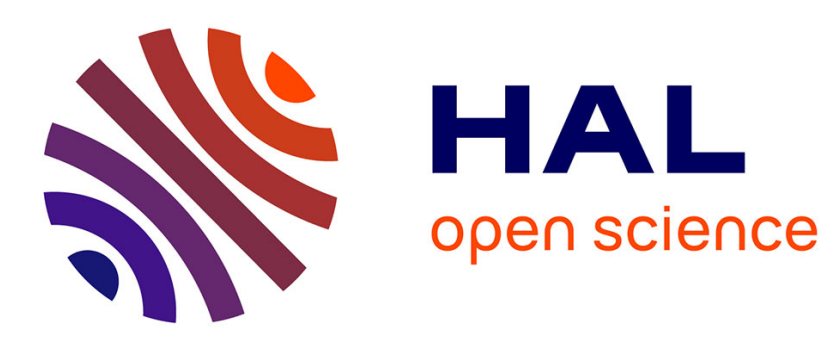

\title{
QoI and Energy-Aware Mobile Sensing Scheme: A Tabu-Search Approach
}

Rim Ben Messaoud, Yacine Ghamri-Doudane

\section{To cite this version:}

Rim Ben Messaoud, Yacine Ghamri-Doudane. QoI and Energy-Aware Mobile Sensing Scheme: A Tabu-Search Approach. IEEE VTC Fall 2015, Sep 2015, Boston, United States. 10.1109/VTCFall.2015.7391101 . hal-01287703

\section{HAL Id: hal-01287703 https://hal.science/hal-01287703}

Submitted on 20 Mar 2016

HAL is a multi-disciplinary open access archive for the deposit and dissemination of scientific research documents, whether they are published or not. The documents may come from teaching and research institutions in France or abroad, or from public or private research centers.
L'archive ouverte pluridisciplinaire HAL, est destinée au dépôt et à la diffusion de documents scientifiques de niveau recherche, publiés ou non, émanant des établissements d'enseignement et de recherche français ou étrangers, des laboratoires publics ou privés. 


\title{
QoI and Energy-aware Mobile Sensing Scheme: A Tabu-Search approach
}

\author{
Rim Ben Messaoud*† and Yacine Ghamri-Doudane ${ }^{\dagger}$ \\ *University of Paris-Est, Gaspard Monge Computer Science Laboratory (LIGM), Champs sur Marne, France \\ ${ }^{\dagger}$ University of La Rochelle, Laboratory of Informatics, Image and Interaction (L3I), La Rochelle, France \\ \{rim.ben_messaoud, yacine.ghamri\}@univ-lr.fr
}

\begin{abstract}
Mobile phones equipped with a rich set of embedded sensors enhance participatory sensing to collect data for different applications. However, many challenges arise when selecting participants to perform sensing tasks. Among these challenges, we can cite energy consumption, users' mobility impact and the quality of retrieved data, recently defined as Quality of Information (QoI). In this work, we study the QoI and Energy-aware Mobile Sensing (QEMSS) problem. Hence, for a given set of users, a sensing area and data quality requirements, the objective of QEMSS is to find the subset of users that maximizes QoI in terms of spatial and temporal metrics while minimizing the overall energy consumption and reducing the redundancy during the sensing process. We propose a meta-heuristic algorithm based on Tabu-Search to provide a sub-optimal solution. Simulation results, for both deterministic and unknown participants' trajectories, are compared to other state-of-the-art methods. This allows showing that our approach outperforms both the greedy-based and the random selection strategies. Particularly, the achieved data quality by our scheme is significantly higher in challenging scenarios such as low dense areas or scarce users' energy resources.
\end{abstract}

\section{INTRODUCTION}

Mobile devices nowadays are equipped with numerous embedded sensors such as accelerometer, GPS, gyroscope, camera and microphone [1] to cite a few, besides their communication, processing and storage capacities. This enhances their role in collecting and sharing data by the crowd (people), a trend called Crowdsensing. Participatory sensing, or Crowdsensing, was first introduced by authors in [2]. Recently, various applications are enabled by this new paradigm of mobile sensing. For example, by leveraging the sensors in users' handsets, location data can be collected to estimate the personalized environmental impact [3]. Other promising applications have been developed in different fields: health care, tourism and transportation [4].

However, these systems raise new challenges compared to traditional WSNs. Authors in [5] pointed out the security issue and built a framework, called Anonysense, to protect the privacy of participants in large scale mobile sensing applications. Another major focus was the fact that smart devices are energy-constrained which requires specific selection schemes to reduce the dedicated energy for sensing tasks. In [6], authors proposed various selection algorithms to show potential energy savings by sensing in a collaborative way. To this purpose, only a subset of participants is selected to cover the area targeting the minimization of the overall energy consumption. Furthermore, authors in [7] proposed collecting "readings" cooperatively and periodically by mobile phones to be mapped into virtual stationary sensors and used later by applications tiers which limits energy consumption during sensing tasks.

In spite of the benefits that may be brought by such a new paradigm, participants may be reluctant to contribute their resources during the sensing procedure. Motivation mechanisms, denoted as "incentives", were introduced to stimulate users to dedicate a percentage of their devices' energy. The MCARD algorithm illustrated in [8] targets selecting participants to get all the required data such that the total cost on paying them is minimized.

One element that hadn't been well addressed in previous works is the quality of collected data, which should be a major requirement for sensing applications. Quality of Information (QoI) was first studied by Bisdikan et al. for WSNs [9] and it has been recently investigated for Crowdsensing. Authors in [10] studied the QoI issue while providing incentives for selected participants. The aforementioned scheme proposed a multi-task oriented mobile users selection strategy (DPS) to maximize a QoI-satisfaction metric per sensing task subject to a budget constraint, yet, energy efficiency was handled very partially.

With all this in mind, we propose a QoI and Energy-aware Mobile Sensing Scheme, (QEMSS), for large scale participatory sensing. The objective is to maximize the overall achieved quality of data while ensuring no-redundancy and respecting energy-constraints towards registered participants. We formally model the corresponding optimization problem and propose a Tabu-Search based algorithm to reach a sub-optimal solution. The effectiveness of our strategy was extensively evaluated in terms of Maximum Achieved QoI and Spatial and Temporal Accuracy. Eventhough results for high density sensing areas are comparable to other selection schemes in the literature, the importance of our work is demonstrated for low density sensing regions and its limited requirements in terms of users' resources.

The rest of the paper is organized as follows. We start by explaining the techniques used in this work: i.e. the TabuSearch meta-heurstic, in section II. Next, we detail the system model and define necessary elements to model the corresponding optimization problem in section III. In section IV, we describe in details the use of Tabu-Search for designing our solution QEMSS. Section V illustrates our simulation results while comparing QEMSS to the two concurrent methods: DPS and Random Selection algorithms. Finally, conclusions and future perspectives are drawn in section VI. 


\section{BACKGROUND: TABU-SEARCH}

Tabu search is a meta-heuristic that guides a local heuristic search procedure to explore the solution space beyond local optimality [11]. It was first introduced by Glover in [12]. The basic idea is to forbid a move that would return to recently visited solutions called tabus.

Given $S$, the set of possible solutions to a problem, for each solution $s \in S$ it exists a subset of $S$ called neighborhood of $s, N(s)$. The neighborhood contains feasible solutions, each is obtained by making a simple move from the solution $s$. A move $m$ can be defined as adding/deleting an element to/from the current solution. Besides, the Tabu Search (TS) algorithm uses a memory structure called Tabu List (TL) to avoid cycles. A solution among $N(s)$ is selected only if it does not exist in the TL. At each iteration, the TS updates the TL by adding attributes of the selected solution. Attributes usually do not contain the complete solution to facilitate handling the TL. The TL size is called Tabu Tenure (TT). It is crucial to define an adequate TT, because if it is too small, there is a high chance to have cycles and hence TS cannot go beyond the local optimal solution. However, if TT is very large, very few options will be left for the neighborhood formation.

To start TS, an Initialization phase is set. This is to generate an initial feasible solution $s_{\text {init }}$. Note that the further is this solution from the optimal one, the greater is the overall time of execution of the method. Therefore, it would be more efficient not to start with a totally random solution. The use of this search in designing our solution, QEMSS, is presented in details in section IV.

\section{System Model AND PROBlem FORMUlation}

\section{A. System Model}

We consider a system of $N$ mobile users and a central server as shown in Figure 1. Each user carries his mobile equipped with embedded sensors and is considered as a candidate participant for the sensing task. Users are moving arbitrarily in a $2 D$ area representing a city. Nevertheless, they do not move in a totally random way but rather they follow paths. The trajectory of each user can be then formally defined as a set of possible visited areas ("locations"). In this case, we subdivide the sensing region into multiple sub-regions of one or many locations.

First, the server initiates the sensing tasks with a set of QoI requirements: $q_{i} \in\left\{q_{1}, \ldots, q_{L}\right\}$, such as quantity of collected data, sensing region and time of measurements. The server regularly gathers information from registered mobile phones users regarding their mobility. Moreover, each participant is assumed to update his device remaining energy. In our scenario, we assume that the unit energy consumption of a sensor on different type of devices is almost identical as assumed by the authors in [13] and we denote it by $e_{c}$. It is worth mentioning that we consider also a threshold level on energy $e_{\theta}$. Whenever a device's energy level reaches this value, the user in question is eliminated from the candidate list of participants during future sensing periods. This guarantees to participants the necessary energy for normal use of their mobiles. The server selects then the subset of participants that maximizes the QoI of overall collected data while taking

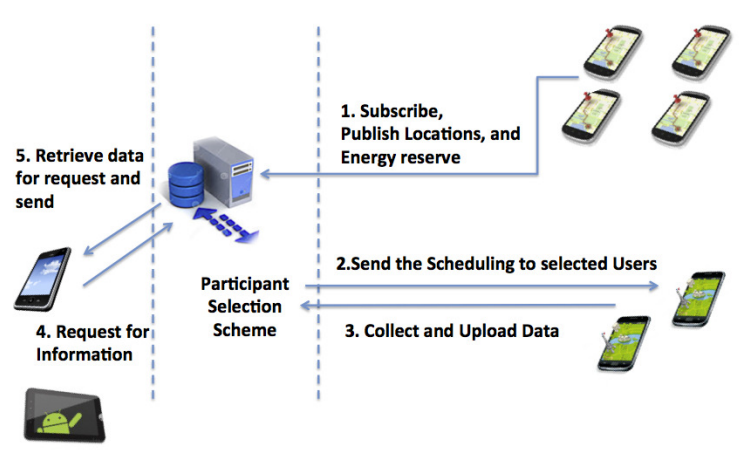

Fig. 1. Mobile Phone Sensing scenario: the server selects a subset of users that answers the maximum QoI while considering an energy constraint and broadcasts the scheduling. Selected participants collect and upload the data. The server answers requests generated by application tiers by retrieving the adequate information.

into account energy and redundancy. To do so, we limit the number of required measurements to one measurement per area for a particular sensing period. Finally, a sensing schedule is generated to determine when and where to sense for each participant.

\section{B. Problem Formulation}

1) QoI attributes and metrics: In the following, we use the notation from TABLE I. Suppose that $X$ is the subset of the selected users among all participants. We compute, for each user, the possible provided data quality. To this point, we selected three attributes: "Completeness", "Timeliness" and "Affordability". The first term measures if the collected data provides all facts. In this work, the spatial completeness is considered as the area potentially covered by a participant. Timeliness is, as the term indicates, collecting and uploading information within a limited time interval which is the sensing period. The last criterion highlights the fact that a user with a low battery level cannot "afford" to participate in next sensing tasks. We quantify the aforementioned attributes using the utility function introduced in [14] as a metric. This normalized function was proposed in economy to quantify the level of usefulness that a user derives from a given product. In our scenario, we set different functions to measure the utility perceived by the system of each QoI attribute .

According to [14], the "completeness" metric is an upward criterion, as the more a user covers an area, the more he collects samples of data. Given a variation range of the coverage $x, x_{\alpha} \leq x \leq x_{\beta}$ and a middle point of the utility $x_{m}$, an adequate utility function for $x$ is:

$$
U_{c}(x)= \begin{cases}0 & x<x_{\alpha} \\ \frac{\left(\frac{x-x_{\alpha}}{x_{m}-x_{\alpha}}\right)^{\zeta}}{1+\left(\frac{x-x_{\alpha}}{x_{m}-x_{\alpha}}\right)^{\zeta}} & x_{\alpha} \leq x \leq x_{m} \\ 1-\frac{\left(\frac{x_{\beta}-x}{x_{\beta}-x_{m}}\right)^{\gamma}}{1+\left(\frac{x_{\beta}-x}{x_{\beta}-x_{m}}\right)^{\gamma}} & x_{m}<x \leq x_{\beta} \\ 1 & x>x_{\beta},\end{cases}
$$

where

$$
\zeta \geq \max \left\{\frac{2\left(x_{m}-x_{\alpha}\right)}{x_{\beta}-x_{m}}, 2\right\}
$$


and

$$
\gamma=\frac{\zeta\left(x_{\beta}-x_{m}\right)}{x_{m}-x_{\alpha}} .
$$

$\zeta$ and $\gamma$ are the tuned steepness parameters.

As per the "Timeliness" metric, it must be a centered function, because the sensing period is a time interval with the required instant of measurement as the median. This ensures that as the time of measurement gets further from the required moment, its utility lowers. Given a variation range of the instant $x, x_{\alpha} \leq x \leq x_{\beta}$ and the required instant of measurement $x_{r}$ as a middle point of the utility, we formulate the utility function described in Equation (4).

$$
U_{t}(x)= \begin{cases}0 & x<x_{\alpha}, x>x_{\beta} \text { (4a) } \\ \frac{\left(\frac{x-x_{\alpha}}{x_{m 1}-x_{\alpha}}\right)^{\zeta}}{1+\left(\frac{x-x_{\alpha}}{x_{m 1}-x_{\alpha}}\right)^{\zeta}} & x_{\alpha} \leq x \leq x_{m 1} \quad \text { (4b) } \\ 1-\frac{\left(\frac{x_{r}-x}{x_{r}-x_{m 1}}\right)^{\gamma}}{1+\left(\frac{x_{r}-x}{x_{r}-x_{m 1}}\right)^{\gamma}} & x_{m 1}<x<x_{r} \quad \text { (4c) } \\ 1 \quad & x=x_{r} \quad \text { (4d) } \\ 1-\frac{\left(\frac{x-x_{r}}{x_{m 2}-x_{r}}\right)^{\zeta}}{1+\left(\frac{x-x_{r}}{x_{m 2}-x_{r}}\right)^{\zeta}} & x_{r}<x \leq x_{m 2} \quad \text { (4e) } \\ \frac{\left(\frac{x_{\beta}-x}{x_{\beta}-x_{m 2}}\right)^{\gamma}}{1+\left(\frac{x_{\beta}-x}{x_{\beta}-x_{m 2}}\right)^{\gamma}} & x_{m 2}<x \leq x_{\beta} \quad \text { (4f) }\end{cases}
$$

where $x_{m 1}$ and $x_{m 2}$ are respectively the middle points of the time intervals $\left[x_{\alpha}, x_{r}\right]$ and $\left[x_{r}, x_{\beta}\right]$.

Finally, the total utility of data is the product of all its QoIattributes utilities presented by Equation (5). This formulation makes the collected information "utile" only if it satisfies all quality metrics. Hence, in case one quantity is null, the overall data-utility provided by the corresponding mobile user in a specific area is null which forbids, for example, collecting data out of the sensing time interval.

$$
U_{Q o I}=U_{c}(x) \times U_{t}(x) .
$$

Equation (5) illustrates the objective function to be maximized in our scheme: i.e. we target to maximize the "timeliness" and "completeness" of collected data during sensing tasks. For the "affordability" attribute, its corresponding metric will be introduced as a constraint in our problem rather than an objective since it is a limiting condition. Besides, the "affordability" is a downward criterion; when a user's mobile remaining energy gets low, the utility of its measurements lowers as well. Hence, we quantify the "affordability", i.e. the capability of a mobile to sense, by the decreasing function of Equation (6).

$$
U_{e}(x)=1-U_{c}(x) .
$$

Note that the percentage of energy $x$ varies in $\left[e_{\theta}, 100 \%\right]$ where $U_{e}\left(e_{\theta}\right)=0$.

2) Known/Unknown users' trajectories: It is worth noting that we consider both deterministic and unknown users' trajectories. The first approach assumes that the server knows in advance each user's trip. This helps to define a benchmark for the unknown case: i.e. the realistic scenario where users announce only their initial locations obtained via the GPS service to the server at the beginning of each sensing period.
TABLE I. LIST OF NOTATIONS

\begin{tabular}{|l|c|}
\hline Notations & Description \\
\hline$N$ & Set of candidate participants \\
\hline$M$ & Sensing sub-regions \\
\hline$L$ & Number of QoI requirements \\
\hline$e_{i}$ & Initial Energy level for user $i$ \\
\hline$e_{c}$ & Energy cost for sensing \\
\hline$e_{\theta}$ & Energy level Threshold \\
\hline$q_{i}$ & A QoI requirement \\
\hline$P_{i n i t}$ & Initial participant position \\
\hline$U_{Q o I}$ & Total QoI-Utility achieved by a user \\
\hline$X$ & Subset of selected users \\
\hline
\end{tabular}

To predict next possible visited areas, we use the Markov model for mobility prediction. Particularly, we choose the second-order Markov model, that is to predict the next position based on the two last visited ones. To this end, we define the probability of visiting a future area as introduced in [15] by:

$$
p_{r s}=\frac{\left|T_{i} /\left(n_{r}, n_{s}\right) \in T_{i}\right|}{\left|T_{i} / n_{r} \in T_{i}\right|},
$$

where $n_{r}$ is the last two visited positions vector, $n_{s}$ is the possible future sub-area to be visited and $T_{i}$ is the trajectory of the user $i$.

3) Optimization Problem: The final goal of QEMSS is to maximize the overall achieved value of data quality collected by $X$ selected participants while minimizing the consumed resources to achieve that. Hence, an optimization problem can be formulated as:

$$
\begin{array}{lll}
\underset{x}{\operatorname{maximize}} & \sum_{i=1}^{N} \sum_{j=1}^{M} x_{i j} \times U_{Q o I}\left(Q o I_{i j}\right) & \\
\text { subject to } & U_{e}\left(e_{i}-e_{c} \sum_{j=1}^{M} x_{i j}\right) \geq 0 & \forall i \in 1 \ldots N \\
\text { and } & \sum_{i=1}^{N} x_{i j} \leq 1 & \forall j \in 1 \ldots M
\end{array}
$$

where $X$ is a users $\times$ areas sized-matrix with elements in $\{0,1\}$. If $x_{i j}$ is 1 , user $i$ is selected to sense on area $j$. The first constraint ensures to each mobile the necessary amount of energy for the common activities while the second one forbids data redundancy by selecting at most one user per area.

In the formulation in (8), we look for a "combination" of users that satisfies both constraints and maximizes the objective function. However, combinatorial optimization problems are challenging to solve whenever a large number of entities is involved. In this paper, we are working on a large scale Crowdsensing scenario which makes looking for the optimal solution a non polynomial-time process [10]. Therefore, it would be more efficient to search a sub-optimal solution using heuristic methods such as Tabu-Search (TS).

\section{QoI and Energy-aware mobile Sensing Scheme}

In the following, we present in details the use of TabuSearch for designing our solution QEMSS. First, we start with defining the different components of TS:

- A possible solution $X$ is a users $\times$ areas sized boolean matrix: i.e. $x_{i j}$ in $\{0,1\}$. This solution must answer the constraints of the problem in Equation (8). 
That means, for each user $\left(u_{i}\right.$ : row of $\left.X\right)$ the remaining energy of his mobile device, $e_{i}-e_{c} \sum_{j=1}^{M} x_{i j}$, needs to be above the defined threshold $e_{\theta}$. Besides, to have at most a user per sub-area, the sum of each column $a_{j}$ of the feasible solution must be less than or equal to 1 to avoid redundancy.

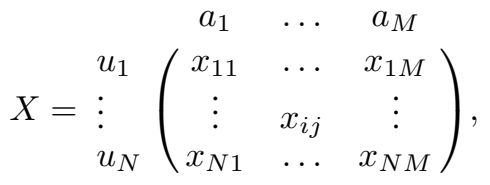

- A move $m \in M(X)$ is a modification applied to an initial solution $X_{\text {init }}$ to generate other possible solutions. In our work, we consider swapping the assignment of users to a same area. Thus, a move $m$ can be a matrix of the same size as $X$ with all elements equals to zeros except those of the old and the new assignment positions, which are set to 1 .

- To generate the neighborhood of a solution $X$, we apply the defined move $m$. Each element in $N(X)$ is the result of a simple XOR operation: $X^{\prime}=X \oplus m$.

- For each solution $X \in S$, we compute the maximum achieved QoI value as its attribute.

- In each iteration, we update the TL by adding the attributes of the best achieved solution $X_{\text {best }}$ in terms of the maximum achieved QoI.

We add the best achieved QoI-value among all visited solutions to the TL as an attribute of $X$ such as done in [11]. This will not only forbid recycling to already visited solutions but also to not visit solutions with same $U_{Q o I}$. Consequently, we reduce both the time of computation and the required memory for TL. Our TS-based selection scheme is presented in Algorithm 1.

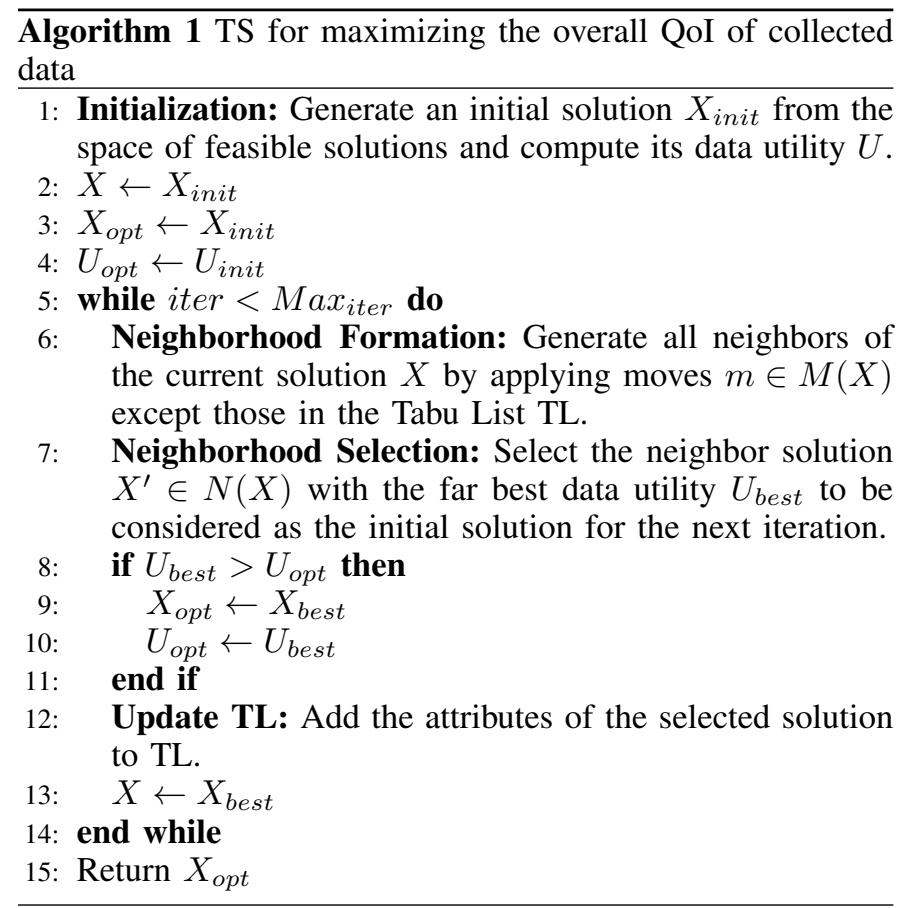

Initialization: To generate an initial solution, we need to satisfy all constraints of the optimization problem (8). A first trivial solution may be the identity matrix which answers the second constraint: at most one user per area. However, note that we can not assign a user to an area which does not belong to his trajectory. To this end, we propose a simple heuristic to find an initial feasible solution. It is a "greedybased procedure" presented in Algorithm 2. First, we select a random sub-region among the set of all sensing sub-regions. Then, we search for the participant with the maximum value of QoI and a residual mobile energy above the defined threshold. We repeat this procedure till we cover all the required area or no more candidates are available.

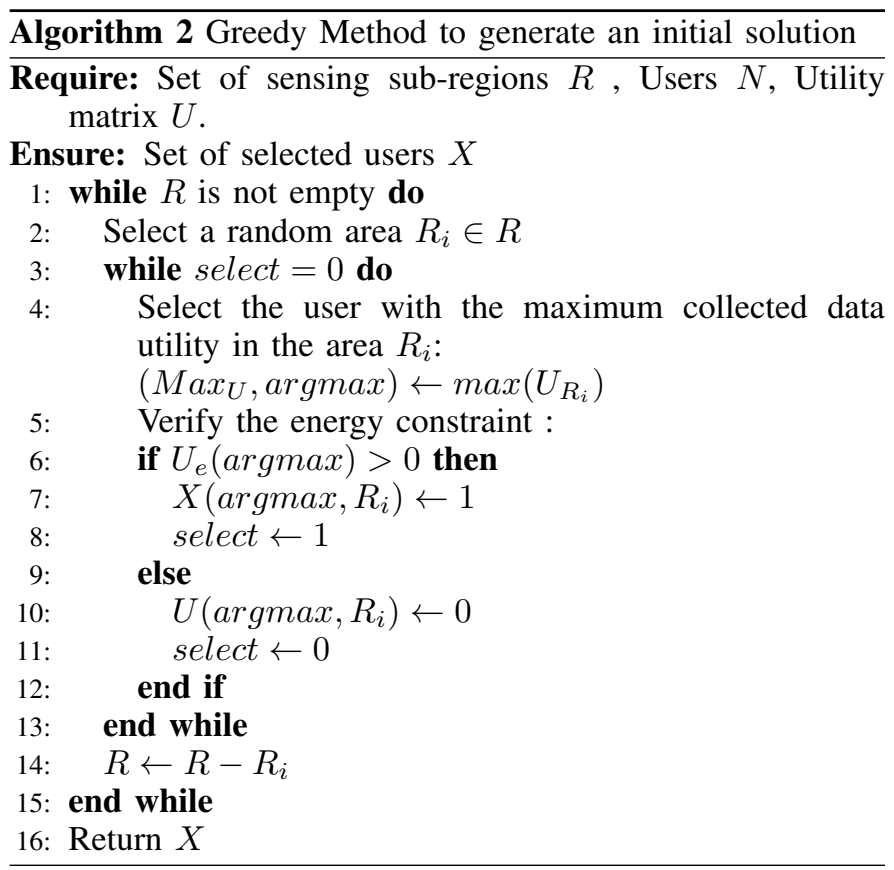

Neighborhood Formation: Starting from a solution $X$ we can generate the neighborhood $N(X)$ by applying one move. We swap a selected user to sense on a specific area in $X$ with another user in the same area. Our swap is based on a generated reference matrix $X_{r e f}$, where $x_{r e f}(i, j)=1$ only if the user $i$ stopped by the area $j$ during his trip. All moves are chosen among the 1-elements of the current region (column of the matrix). By this way, we obtain a "Reduced Neighborhood" which facilitates the search among feasible solutions. As for the initial solution, we check that each neighbor $X^{\prime} \in N(X)$ conforms with the energy constraint.

Note that Algorithm 1 is the search strategy for both deterministic and unknown trajectories approaches. Simulations are described in detail in the next section and results are compared to existing concurrent participatory sensing schemes from the literature.

\section{PERFormance Evaluation}

We consider as a simulation area, a city of $4000 \mathrm{~m} \times 6000 \mathrm{~m}$ scale which includes 20 horizontal "roads" and 20 vertical ones. We first subdivide this area into sub-areas of "roads". The topology of the area is similar to the well known Manhattancity model. Therefore, we use mobility traces of the Manhattan 


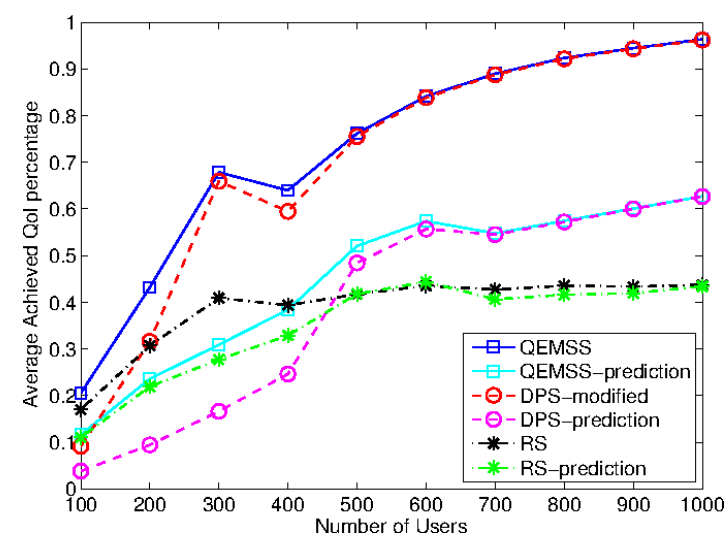

(a) Average achieved QoI percentage for different number of users, for both deterministic and unknown trajectories cases

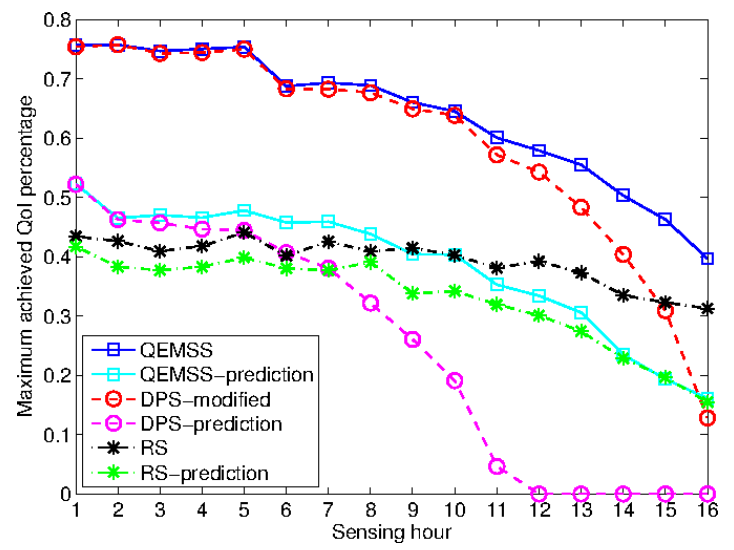

(b) Maximum achieved QoI percentage by 400 users during a 16-hours sensing day, for both deterministic and unknown trajectories cases

Fig. 2. Achieved QoI by different selection schemes in case of known/unknown trajectories while (a) varying the number of users in the sensing area and (b) considering the energy evolution for 400 users during a 16-hour sensing period.

mobility model generated by the mobility scenario generation tool, Bonnmotion [16]. Each user enters a road with a random speed picked from $[1,3]$, moves straight with a probability of 0.5 , turns left or right with respective probabilities of 0.25 each. The number of users is varied from 100 to 1000 . Users' initial energy is randomly generated as a uniformlydistributed random variable ranging from $e_{\theta}$ to $100 \%$. We carried out simulations for 16-hours sensing period. Besides, we conducted two predictions per hour for the unknown trajectories scenario.

To evaluate the performance of our algorithm, we introduce three metrics: Maximum Achieved QoI, Spatial Accuracy and Temporal Accuracy. QEMSS was compared to two methods, a modified version of the Dynamic Participant Selection Scheme (DPS) algorithm in [10] and a Random selection scheme. Results are detailed in Figures 2 and 3.

\section{A. Maximum Achieved QoI}

In Figure 2, we plot the achieved data quality percentage achieved by the three considered selection schemes. The average value of the different 16-hours measured QoI percentage varying the number of users is shown in Figure 2(a).

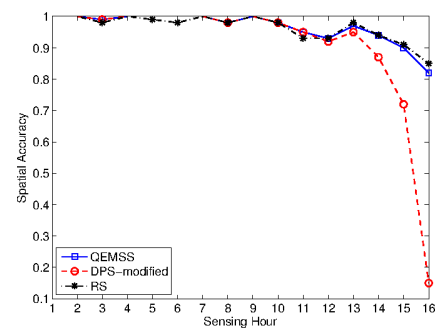

(a) Spatial accuracy - 400 users

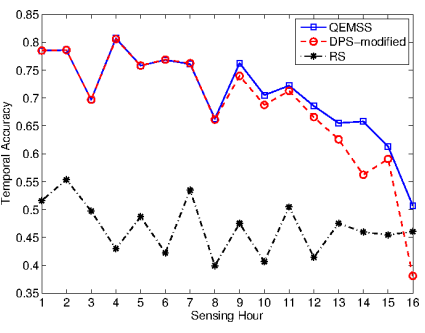

(c) Temporal accuracy - 400 users

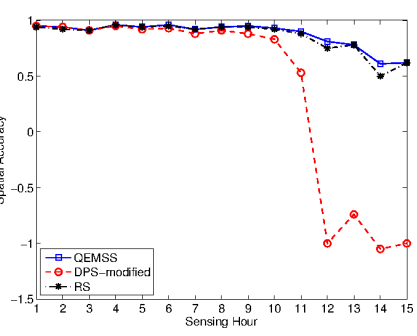

(b) Spatial accuracy - 200 users

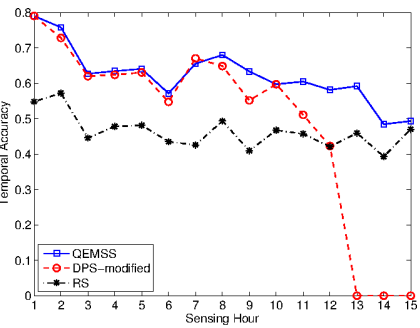

(d) Temporal accuracy - 200 users
Fig. 3. Data Accuracy in spatial (a), (b) and temporal (c), (d) terms for different sensing region density (200-400) users

Obviously, the reached QoI value increases in function of the number of users available in the sensing area. For high density areas (500-1000 users), QEMSS performs as efficient as others schemes. However, for low density (100-400 users), our scheme outperforms both DPS and RS. This is observed for both deterministic and prediction algorithms. The maximum achieved QoI value, in the case of unknown trajectories, gets lower for all algorithms due to the prediction resulted errors. Nevertheless, QEMSS still performs better than the two other evaluated schemes.

In Figure 2(b), we observe that for a specific number of users (400) the performance of our scheme is comparable to the DPS algorithm in the beginning of the period. Nevertheless, the gap between QEMSS and DPS widened in the end of the sensing period up to $27 \%$. This is due to the selection strategy; the TS-based algorithm used by QEMSS conducts extensive search at each sensing interval to diversify the selected users which makes their devices' batteries last more. However, using the DPS greedy-based search, the same users are frequently selected to sense which limits their resources. The Random Selection has clearly the lower performance in terms of achieved QoI.

\section{B. Spatial and Temporal Accuracy}

To measure the spatial accuracy, we used the Manhattandistance function as presented by Equation (9). We compute the distance between the spatial coordinates of the required sensing area and the collected data. Note that the server answers applications' requests by available data, i.e, if no measurement is collected on the required area, it looks for the spatially and temporally closest measurement. That is, the larger is the Manhattan-distance $\operatorname{dist}\left(\right.$ area $_{\text {req }}$, data $\left._{\text {coll }}\right)$, the lower is its spatial accuracy.

$$
s_{a}=1-\sum_{i=1}^{n}\left|X_{i}-Y_{i}\right|,
$$


As shown in Figure 3(a) and 3(b), the DPS-scheme is getting less accurate when getting closer to the end of the sensing period. This corresponds to the fact of not achieving good data quality. QEMSS and Random Search reached comparable spatial accuracy since they both cover most of the required regions. However, for the timeliness criterion quantified by the utility function $U_{t}(x)$, we observe in Figures 3(c) and 3(d) that the RS-scheme is the less accurate selection strategy. That means, selected users by RS covered most of the area but collected samples in random instants that are far from the required time of measurement. Eventhough DPS is as accurate as QEMSS for the first half of the sensing period, the error rate is leveled up to $62 \%$ by the end of the sensing period. This is due to the limited energy resources of available users at the end of the sensing period when using DPS.

\section{CONCLUSIONS}

In this paper, a QoI Energy-aware Selection Scheme for participatory sensing systems is presented. We investigated first how to quantify different QoI attributes by introducing an adequate "utility function" to each criterion. In order to select an optimal set of users that maximizes data quality, a TabuSearch based algorithm was illustrated. Simulation results show that our system has achieved competitive performance in high density areas compared to other benchmark schemes. However, QEMSS obtained a significant gain in the achieved QoI and spatial and temporal accuracy of data in challenging situations such as low density sensing areas and/or low battery equipped participants at the end of a sensing period. Hence, QEMSS outperformed the two state-of-the-art participatory selection schemes.

Besides, QEMSS diversified the selection of users in each iteration by using the Tabu-search. This resulted on a first level of fairness among participants. A future work will study in detail the fairness issue in order to reduce the individual energy consumption by minimizing the number of sensing times for each participant.

\section{REFERENCES}

[1] N. Lane, E. Miluzzo, H. Lu, D. Peebles, T. Choudhury, and A. Campbell, "A survey of mobile phone sensing," IEEE Communications Magazine, vol. 48, no. 9, pp. 140-150, 2010.

[2] J. Burke, D. Estrin, M. Hansen, A. Parker, N. Ramanathan, S. Reddy, and M. B. Srivastava, "Participatory sensing," in WSW: Mobile Device Centric Sensor Networks and Applications, 2006, pp. 117-134.

[3] M. Mun, S. Reddy, K. Shilton, N. Yau, J. Burke, D. Estrin, M. Hansen, E. Howard, R. West, and P. Boda, "PEIR, the personal environmental impact report, as a platform for participatory sensing systems research," in Mobile Systems, Applications and Services, 2009, pp. 55-68.

[4] P. Mohan, V. N. Padmanabhan, and R. Ramjee, "Nericell: Rich monitoring of road and traffic conditions using mobile smartphones," in ACM conf. on Embedded Network Sensor Systems, 2008, pp. 323-336.

[5] C. Cornelius, A. Kapadia, D. Kotz, D. Peebles, M. Shin, and N. Triandopoulos, "AnonySense: Privacy-aware people-centric sensing," in $\mathrm{Mo}$ bile Systems, Applications and Services, 2008, pp. 211-224.

[6] X. Sheng, J. Tang, and W. Zhang, "Energy-efficient collaborative sensing with mobile phones," in INFOCOM, 2012, pp. 1916-1924.

[7] H. Weinschrott, F. Durr, and K. Rothermel, "Streamshaper: Coordination algorithms for participatory mobile urban sensing," in Mobile Adhoc and Sensor Systems (MASS), 2010, pp. 195-204.

[8] X. Lu, D. Li, B. Xu, W. Chen, and Z. Ding, "Minimum cost collaborative sensing network with mobile phones," in International Conference on Communications (ICC), 2013, pp. 1816-1820.
[9] C. Bisdikian, J. Branch, K. Leung, and R. Young, "A letter soup for the quality of information in sensor networks," in Pervasive Computing and Communications (PerCom), 2009, pp. 1-6.

[10] Z. Song, C. Liu, J. Wu, J. Ma, and W. Wang, "QoI-aware multitaskoriented dynamic participant selection with budget constraints," IEEE Transactions on Vehicular Technology, vol. 63, no. 9, pp. 4618-4632, 2014.

[11] H. Kamal, M. Coupechoux, and P. Godlewski, "A tabu search DSA algorithm for reward maximization in cellular networks," in Wireless and Mobile Computing, Networking and Communications (WiMob), 2010, pp. 40-45.

[12] F. Glover, "Tabu search-Part I," ORSA Journal on Computing, vol. 1, no. 3, pp. 190-206, 1989.

[13] I. Koenig, A. Q. Memon, and K. David, "Energy consumption of the sensors of smartphones," in Int. Symposium on Wireless Communication Systems (ISWCS), 2013, pp. 1-5.

[14] Q.-T. Nguyen-Vuong, Y. Ghamri-Doudane, and N. Agoulmine, "On utility models for access network selection in wireless heterogeneous networks," in IEEE Network Operations and Management Symposium, NOMS, 2008, pp. 144-151.

[15] L. Pournajaf, L. Xiong, and V. Sunderam, "Dynamic data driven crowd sensing task assignment," Procedia Computer Science, vol. 29, no. 0, pp. $1314-1323,2014$.

[16] N. Aschenbruck, R. Ernst, E. Gerhards-Padilla, and M. Schwamborn, "Bonnmotion: a mobility scenario generation and analysis tool," in Int. Conf. on Simulation Tools and Techniques (ICST), 2010. 